\section{THU0332 ABSOLUTE REDUCTION OF REGULATORY T CELLS AND EFFICACY OF SHORT-TERM AND LOW-DOSE IL-2 IN PATIENTS WITH DERMATOMYOSITIS OR POLYMYOSITIS}

Min Feng ${ }^{1}$, Xiang-Cong Zhao ${ }^{1}$, Chong Gao ${ }^{2}$, Jing Luo ${ }^{1} .{ }^{1}$ The Second Hospital of Shanxi Medical University, Rheumatology, Taiyuan, China; ${ }^{2}$ Brigham and Women's Hospital, Harvard Medical School, Pathology, Boston, United States of America

Background: Dermatomyositis (DM) and polymyositis (PM) are heterogeneous, chronic and progressive autoimmune disorders characterized by symmetrical, proximal muscle weakness[1,2]. Although the pathogenesis of PM/DM remains unclear, immune intolerance caused by the deficiency of regulatory $T$ (Treg) cells may be an important cause of PM/DM, and quantitative status of peripheral Treg cells is unclear in this disease. Low-dose interleukin-2 (Id-IL2) has been found to enhance proliferation, function and survival of Treg cells to regulate immune tolerance[3]. However, the therapeutic effect of Id-IL2 on PM/DM has not been reported.

Objectives: To investigate whether peripheral Treg cells are reduced and whether Id-IL2 has therapeutic efficacy in PM/DM.

Methods: Total 71 PM/DM inpatients (10 PM and $61 \mathrm{DM}$ ) were retrospectively studied. Five PM and $35 \mathrm{DM}$ patients were treated with Id-IL2 (5.0*105 international units (IU) per day for 5 days) accompanied by conventional therapy, while the rest PM/DM patients only with conventional therapy. Thirty of age- and gender-matched healthy adults were regarded as healthy controls (HCs). CD3+T, CD4+T, CD8+T, B, NK, Th1, Th2, Th17 and Treg cells, as well as cytokines [interleukin-2 (IL-2), interleukin4 (IL-4), interleukin-6 (IL-6), interleukin-10 (IL-10), tumor necrosis factor- $\alpha$ (TNF- $\alpha$ ), interferon- $\gamma$ (IFN- $\gamma$ ) and interleukin-17 (IL-17)] were measured by flow cytometry. The correlation coefficients between various indicators and Myositis Disease Activity Assessment Visual Analogue Scale (MYOACT) were calculated by Spearman correlation test.

Abstract THU0332 -Table 1.

Table 1. The demographic baseline data of PM/DM patients and HCs

\begin{tabular}{|c|c|c|c|c|}
\hline & $\mathrm{PM}(\mathrm{n}=10)$ & $\operatorname{DM}(\mathrm{n}=61)$ & $\mathrm{HCs}(\mathrm{n}=30)$ & $P$ \\
\hline Age (years) & $47.40 \pm 13.59$ & $54.39 \pm 13.37$ & $53.73 \pm 6.37$ & 0.225 \\
\hline Female/male & 5is & $42: 119$ & $20 / 10$ & 0.521 \\
\hline disease duration (month) & $36.00(20.50,102.00)$ & $6.00(2.00,36.00)$ & - & - \\
\hline WBC $\left({ }^{*} 10^{n} 9 / \mathrm{L}\right)$ & $3.25(5.42,8.20)$ & $7.49(5.50,9.34)$ & $5.83(4.73,6.72)$ & 0.005 \\
\hline NEUT (*10^9/L) & $5.48(3.58,6.74)$ & $5.58(3.49,3.04)$ & $3.28(2.57,3.96)$ & $<0.001$ \\
\hline $\operatorname{LYMP}\left({ }^{*} 10^{\wedge} 9 \mathrm{~L} \mathrm{~L}\right)$ & $0.85(0.45,1.93)$ & $1.15(0.83,1.76)$ & $1.91(1.70,2.27)$ & $<0.001$ \\
\hline $\operatorname{PLT}\left({ }^{*} 10^{n} 9 / \mathrm{L}\right)$ & $282.00(182.25,314.75)$ & $240.00(174.00,306.00)$ & $245.50(218.25,270.25)$ & 0.791 \\
\hline $\mathrm{RDW}(\mathrm{fL})$ & $46.20(43.15,52.10)$ & $44.70(41.88,48.43)$ & $41.30(40.48,43.38)$ & $<0.001$ \\
\hline $\operatorname{ESR}(\mathrm{mm} / \mathrm{h})$ & $73.00(26.25,120.00)$ & $40.00(23.25,31.50)$ & $12.50(6.00,15.00)$ & $<0.001$ \\
\hline $\operatorname{CRP}(\mathrm{mg} \Omega \mathrm{L})$ & $18.95(9.31,74.63)$ & $12.85(5.08,49.55)$ & - & 0.391 \\
\hline $\operatorname{ALT}(\mathrm{UL})$ & $50.10(25.70,63.50)$ & $34.75(16.35,65.95)$ & - & 0.412 \\
\hline $\operatorname{AST}(\mathrm{UL})$ & $35.25(26.35,62.58)$ & $36.30(25.78,72.50)$ & - & 0.880 \\
\hline CK (UR) & $536.50(38.00,2291.50)$ & $54.00(32.77,283.00)$ & - & 0.090 \\
\hline LDH (U/L) & $406.50(324.00,632.63)$ & $338.00(262.00,476.00)$ & - & 0.168 \\
\hline $\mathrm{HBDH}$ (U/L) & $286.50(223.00,527.48)$ & $247.00(195.50,328.50)$ & - & 0.116 \\
\hline anti-ul-SRP & 1 & 4 & - & 1.000 \\
\hline anti-Jo-1 & 0 & 3 & - & 1.000 \\
\hline anti-PM-Scl & 1 & 2 & . & 0.895 \\
\hline anti-Mi-2 & 0 & 1 & . & 1.000 \\
\hline anti-Ku & 1 & 3 & - & 1.000 \\
\hline MYOACT & $4.00(3.00,5.25)$ & $6.00(4.00,7.00)$ & - & . \\
\hline
\end{tabular}

Continuous data were expressed as Mean \pm standard deviation (M $(\mathrm{SD})$ or Median $\left(\mathrm{O}_{25}, \mathrm{Q}_{25}\right)$ and analyzed by one-way analysis of variance (ANOVA) or the Independent-samples Kruskal-Wallis test one-way ANOVA or Mann-Whitney U test. Categorical data were reported as numbers and compared with Chi-square test.

-Abbreviation: WBC: white blood cell; NEUT: neutrophile granulocyte; LYMP: lymphocyte; PLT platelet; RDW: red cell distribution width; ESR: erythrocyte sedimentation rate; CRP: C-reactive protein; ALT: alanine aminotransferase; AST aspartate aminotransferase; CK: creatine kinase, LDH: lactate dehydrogenase; HBDH: hydroxybutyrate dehydrogenase.

Results: Both PM and DM patients had significantly lower number of CD3+T $(P=0.018, \quad P=0.003)$ and $\mathrm{CD} 4+\mathrm{T}$ cells $(P=0.035, P=0.016)$ than $\mathrm{HCs}$, as well as the number and percentage of Treg cells $(P \leq 0.001)$ in PM/DM and these of NK, Th1 cells in DM, while Th2 and Th17 cells didn' $t$ exhibit any significant difference. In addition, the ratio of Th1/Th2 in $\mathrm{DM}(P<0.001)$ and that of Th17/Treg was obviously decreased in $\mathrm{PM} /$
DM $(P=0.015, P=0.001)$. Meanwhile, the production of serum IL-2 was reduced in $\mathrm{PM} / \mathrm{DM}(P \leq 0.001)$, while, in contrast, IL-4, IL-6, IL-10, TNF- $\alpha$, IFN- $\gamma$ and IL-17 increased. Id-IL2 could significantly increase the number of Treg cells but have little effect on Th17 cells in DM. In addition, MYOACT was correlated with the number of $\mathrm{CD} 3+\mathrm{T}, \mathrm{CD} 4+\mathrm{T}, \mathrm{CD} 8+\mathrm{T}$, Th1, Th2, Treg cells, PLT and lymphocyte $(r=-0.388, P=0.002 ; r=-0.360$, $P=0.004 ; \quad r=-0.364, \quad P=0.004 ; \quad r=-0.386, \quad P=0.002 ; \quad r=-0.282, \quad P=0.028 ; \quad r=$ $0.262, P=0.041 ; \mathrm{r}=-0.311, P=0.015 ; \mathrm{r}=-0.388, P=0.002$; respectively) negatively, as well as the percentage of CD3+T and CD8+T cells $(r=-0.277$, $P=0.030 ; \quad r=-0.291, \quad P=0.023$ ), opposite to aspartate transaminase (AST) $(\mathrm{r}=0.289, P=0.025)$ in $\mathrm{DM}$
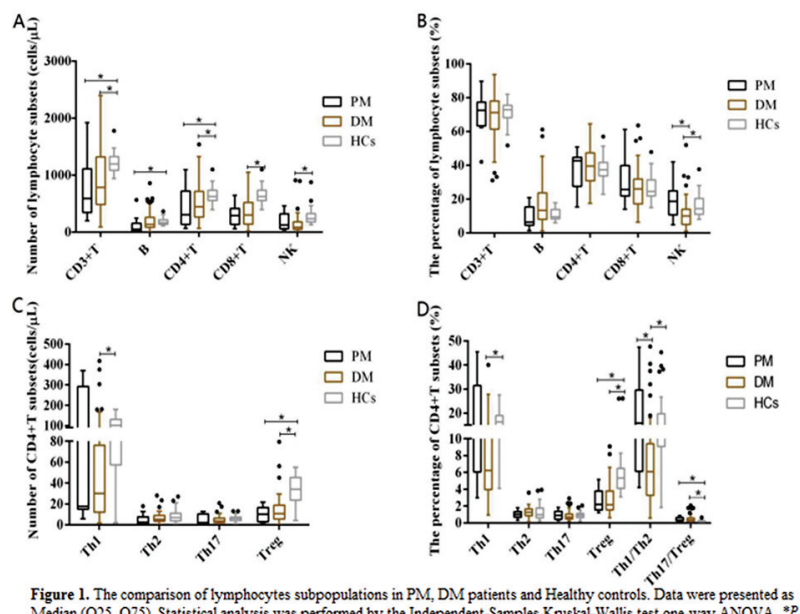
$<0.05$

Abstract THU0332 - Figure 1

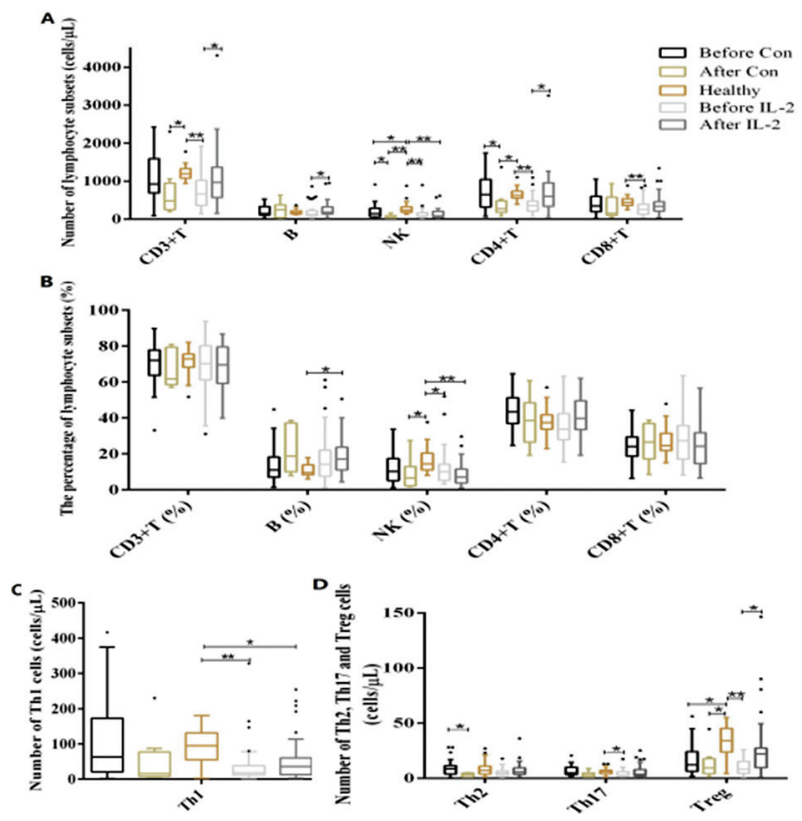

Abstract THU0332 - Figure 2 

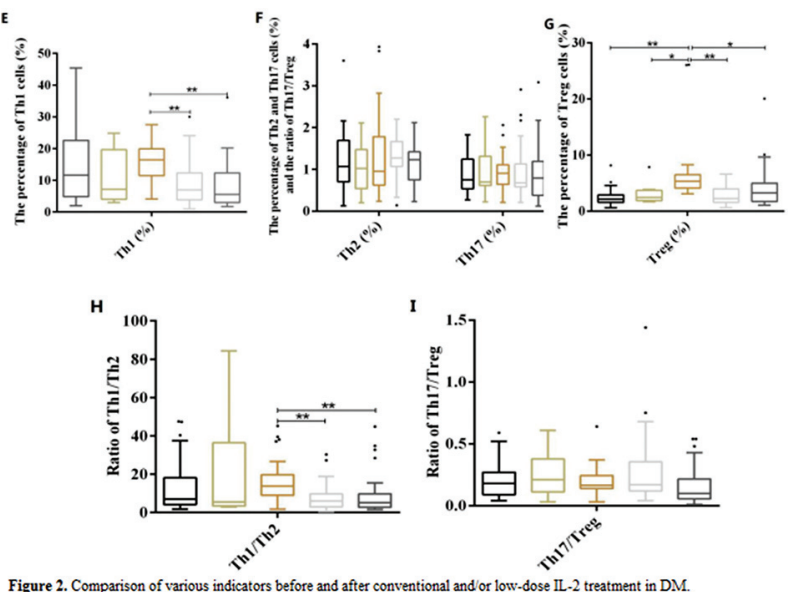

Figure 2. Comparison of various indicators before and after conventional and or low-dose IL-2 treatment in DM.
(A-D) Comparison of lymphocytes subsets and CD4-T cells subsets in healthy controls and DM patients before and after conventional and low-dose IL-2 treatment. Data were expressed as Median $(Q 25, \mathrm{Q} 75)$. Data were analyzed by the Independen Samples Kruskal-Wallis test one-way ANOVA. ${ }^{*} P<0.05 ; * * P<0.001$

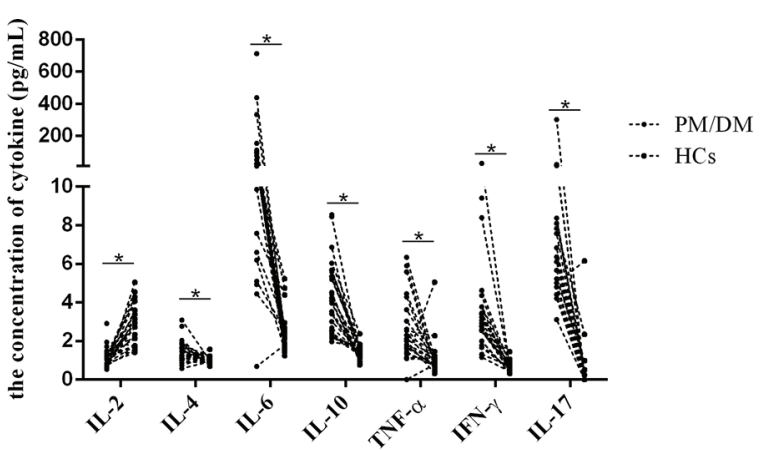

Figure 3. Comparison of serum concentrations of cytokines (including IL-2, IL-4, IL-6, IL10 , TNF- $\alpha$, IFN-g and IL-17) between PM/DM and Healthy Controls by Mann-Whitney U test. $* P<0.05$

Abstract THU0332 - Figure 3

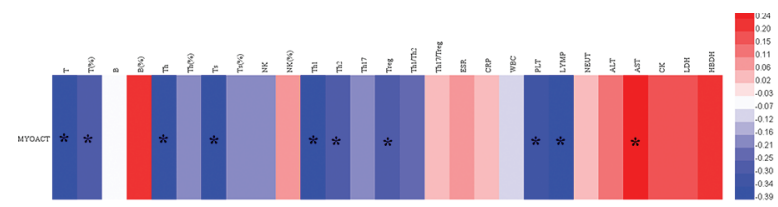

Figure 4 . The correlation between multiple indicators and MYOACT. Heatmap representation of correlation of MYOACT with lymphocyte
subpopulation (including the number and percentage of $C D 3+T, B, T h$, Ts and NK cells), $C D 4+\mathrm{T}$ subsets (including the number of $T$ Th1, Th2, Th17, Treg cells and the ratio of Th1/Th2 and Th17/Treg), Erythrocyle Sedimentation Rate (ESR), C-Reactive Protein (CRP), White Blood Cel (WBC), Platelet (PLT), Lymphocyte (L YMP), Neutrophile Granulocyte (NEUT), Alanine Aminotransferase (ALT), Aspartate Aminotransferase
(AST), Creatine Kinase (CKR), Lactate Dehydrogenase (LDH) and Hydroxybutyrate Dehydrogenase (HBDH) in DM patients ( $(n=61)$. "P $P$ <0.05 by

Abstract THU0332 - Figure 4

Conclusion: The decrease of Treg cells was found in PM/DM accompanied with lower serum IL-2, which should be associated with the pathogenesis of PM/DM. Meanwhile, various pro-inflammatory $T$ cells also showed a downward trend with or without significant difference, indicating $\mathrm{PM} / \mathrm{DM}$ is caused by immunodeficiency rather than hyperimmunism. Id-IL2 combined with conventional therapy could restore the reduction of CD4Treg cells and help to alleviate disease activity.

\section{REFERENCES:}

[1] Yang WM, Chen JJ. Advances in biomarkers for dermatomyositis. Clin Chim Acta 2018; 482: 172-177.

[2] Yuan L, Yao L, Zhao L, Xia L, Shen H, Lu J. Serum levels of soluble ST2 and interleukin-33 in patients with dermatomyositis and polymyositis. Clin Exp Rheumatol 2013; 31:428-432.

[3] Mizui M, Tsokos GC. Low-Dose IL-2 in the Treatment of Lupus. Curr Rheumatol Rep 2016; 18:68.

Disclosure of Interests: None declared DOI: 10.1136/annrheumdis-2019-eular.4022

\section{THU0333 \\ 2-CARBA CYCLIC PHOSPHATIDIC ACID (2CCPA) SUPPRESSES PROFIBROTIC ACTIVITY IN SYSTEMIC SCLEROSIS SKIN FIBROBLASTS AND BLEOMYCIN-} INDUCED SKIN FIBROSIS IN MICE

Tomoaki Higuchi $^{1,1}$, Kae Takagi ${ }^{1}$, Akiko Tochimoto ${ }^{1}$, Yuki Ichimura ${ }^{1}$,

Takanari Norose $^{1}$, Yasuhiro Katsumata ${ }^{1}$, Ikuko Masuda ${ }^{1}$, Hisashi Yamanaka ${ }^{1}$, Toshihiro Moroboshi ${ }^{2}$, Yasushi Kawaguchi ${ }^{1}$. Tokyo Women's Medical University, Department of Rheumatology, Tokyo, Japan; ${ }^{2}$ SANSHO, Co., Ltd, Tokyo, Japan

Background: Systemic sclerosis (SSc) is a connective tissue disorder with progressive fibrosis in multiple organs including skin, lung and the gastrointestinal tract. Fibrosis is thought to be driven by activated fibroblasts. Therefore, inhibition of the profibrotic activity of activated fibroblasts may be a promising therapeutic approach in skin fibrosis in SSc. The autotaxin (ATX)/lysophosphatidic acid (LPA) axis is reportedly involved in fibrotic pathogenesis in $\mathrm{SSc}^{1}$. 2-carba cyclic phosphatidic acid (2ccPA) is a naturally occurring lipid mediator and one of its pleiotropic properties is to inhibit the ATX/LPA axis.

Objectives: We investigated the anti-fibrotic effect of 2ccPA on human SSc skin fibroblasts and bleomycin-induced skin fibrosis in mice. Furthermore, we searched signaling pathways related to the anti-fibrotic effects of 2ccPA.

Methods: This study was approved by the ethics committee and the ethical review committee of animal experiments of Tokyo Women's Medical University. We informed all participants of the content of this study, and written consent was obtained. Skin fibroblasts were taken from SSc patients and adult healthy individuals were purchased. The cells were incubated with 1-10 $\mu \mathrm{M} 2 \mathrm{ccPA}$ in the presence or absence of $10 \mathrm{ng} / \mathrm{ml}$ transforming growth factor- $\beta 1$ (TGF- $\beta 1$ ). Messenger RNA (mRNA) and protein expression for type I collagen, connective tissue growth factor (CTGF), $\alpha$ smooth muscle actin ( $\alpha \mathrm{SMA})$, fibronectin (FN) and TGF- $\beta 1$ were assessed by qRT-PCR or Western blotting. Procollagen type I Cpeptide, prostaglandin $E_{2}\left(P_{G}\right)$ and hepatocyte growth factor (HGF) levels in the supernatant were assessed by ELISA. Intracellular cyclic adenosine monophosphate (CAMP) levels were calculated using a commercially available EIA kit. Forskolin was used to increase intracellular CAMP levels in cultured SSc skin fibroblasts. An inhibitor of adenylate cyclase (AC), 2'-deoxyadenosine, was used to investigate whether the anti-fibrotic effect of 2ccPA was mediated via the AC/cAMP pathway. Furthermore, we used a mouse model of bleomycin-induced skin fibrosis to investigate the safety and anti-fibrotic effects of 2ccPA.

Results: Ten $\mu \mathrm{M}$ 2ccPA significantly reduced mRNA and protein expression for type I collagen, CTGF and $\alpha$ SMA in SSc skin fibroblasts and adult healthy skin fibroblasts treated with TGF- $\beta 1$. PGE2 and HGF levels in the supernatant of SSc skin fibroblasts were also reduced by 2ccPA treatment. 2ccPA increased intracellular cAMP levels as well as the AC stimulator, forskolin. In addition, forskolin decreased the mRNA expression of profibrotic markers. Reduction of COL1A1 mRNA expression by 2cCPA was blocked by treatment with 2'-deoxyadenosine in cultured SSc skin fibroblasts, suggesting that the anti-fibrotic activity of 2ccPA was partially mediated via AC stimulation. In mouse experiments, intraperitoneal injection of $10 \mathrm{mg} / \mathrm{kg} 2 \mathrm{ccPA}$ significantly reduced the development of skin thickness, collagen content and $\alpha$ SMA-positive cell counts.

Conclusion: 2ccPA suppressed the profibrotic activity of SSc skin fibroblasts and the development of bleomycin-induced skin fibrosis. Our experiments suggested that the anti-fibrotic property of 2ccPA was at least in part due to increased intracellular cAMP levels in skin fibroblasts. 2ccPA has been reported to be well tolerated in clinical trials of other diseases and may be expected for the treatment of fibrotic lesions in SSc.

\section{REFERENCE :}

[1] Castelino FV et al. Arthritis Rheumatol. 2016;68:2964-2974.

Disclosure of Interests: Tomoaki Higuchi: None declared, Kae Takagi: None declared, Akiko Tochimoto: None declared, Yuki Ichimura: None declared, Takanari Norose: None declared, Yasuhiro Katsumata: None declared, Ikuko Masuda: None declared, Hisashi Yamanaka Grant/research support from: AbbVie, Eisai, Bristol-Meyers, Novartis, Behringer, Astellas, Kaken, Nippon-Shinyaku, Pfizer, UCB, Ayumi, Ono, Daiichi-Sankyo, Taisyo-Toyama, Takeda, Tanabe-Mitsubishi, Chugai, Teijin Pharma, Torii, YLbio, Speakers bureau: Bristol-Meyers, Astellas, Pfizer, Daiichi-Sankyo, Takeda, Tanabe-Mitsubishi, Chugai, Teijin Pharma, YLbio, Toshihiro Moroboshi: None declared, Yasushi Kawaguchi: None declared DOI: 10.1136/annrheumdis-2019-eular.2637 